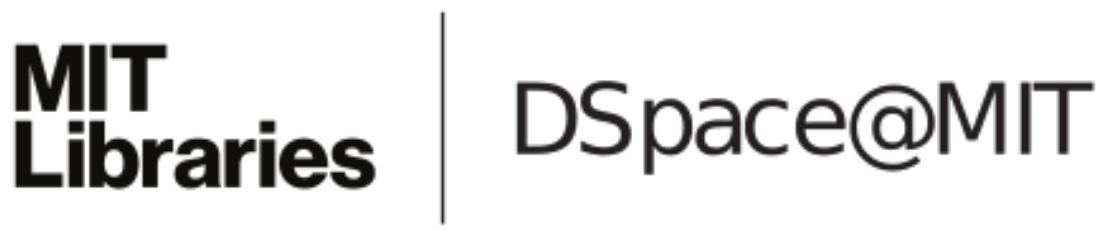

\author{
MIT Open Access Articles
}

Targeting in Advertising Markets:
Implications for Offline Versus Online Media

The MIT Faculty has made this article openly available. Please share how this access benefits you. Your story matters.

Citation: Bergemann, Dirk, and Alessandro Bonatti. "Targeting in Advertising Markets: Implications for Offline Versus Online Media." The RAND Journal of Economics 42.3 (2011): 417443.

As Published: http://dx.doi.org/10.1111/j.1756-2171.2011.00143.x

Publisher: Wiley Blackwell

Persistent URL: http://hdl.handle.net/1721.1/77229

Version: Author's final manuscript: final author's manuscript post peer review, without publisher's formatting or copy editing

Terms of use: Creative Commons Attribution-Noncommercial-Share Alike 3.0 


\title{
Targeting in Advertising Markets: Implications for Offline vs. Online Media*
}

\author{
Dirk Bergemann ${ }^{\dagger} \quad$ Alessandro Bonatti ${ }^{\ddagger}$
}

June 3, 2011

\begin{abstract}
We develop a model with many advertisers (products) and many advertising markets (media). Each advertiser sells to a different segment of consumers, and each medium is targeting a different audience. We characterize the competitive equilibrium in the advertising markets and evaluate the implications of targeting.

An increase in targeting leads to an increase in the total number of consumerproduct matches, and hence in the social value of advertising. Yet, targeting also increases the concentration of firms advertising in each market. Surprisingly, we then find that the equilibrium price of advertisements is first increasing, then decreasing in the targeting capacity.

We trace out the implications of targeting for competing media. We distinguish offline and online media by their targeting ability: low versus high. As consumers' relative exposure to online media increases, the revenues of offline media decrease, even though the price of advertising might increase.

Keywords: Targeting, Advertising, Display Advertising, Sponsored Search, Media Markets.

JEL Classification: D44, D82, D83.
\end{abstract}

${ }^{*}$ The first author gratefully acknowledges financial support from NSF SES 0851200. We wish to thank the Co-Editor, Mark Armstrong, and three anonymous referees for many suggestions that greatly improved the paper. We thank Glenn Ellison, Justin Johnson, Jon Kleinberg, Nancy Lutz, Steven Matthews, Catherine Tucker, Miguel Villas-Boas, Rakesh Vohra, Glen Weyl, and Feng Zhu for helpful comments and discussions. We benefitted from discussions at seminars and conferences at Cornell, CUNY, FTC, MIT, Northwestern, Rochester, Stanford, UBC, UCL, and WISE 2009.

${ }^{\dagger}$ Department of Economics, Yale University, New Haven, CT 06520, dirk.bergemann@yale.edu

${ }^{\ddagger}$ MIT Sloan School of Management, Cambridge, MA 02142, bonatti@mit.edu 


\section{Introduction}

Over the past decade the internet has become an increasingly important medium for advertising. The arrival of the internet has had important consequences on the market position of many traditional media, i.e. offline media such as print, audio and television. For some of these media, most notably the daily newspapers, the very business model is under the threat of extinction due to competition from the internet for the placement of advertising. The following chart shows the recent changes in aggregate spending for advertising on different media. ${ }^{1}$

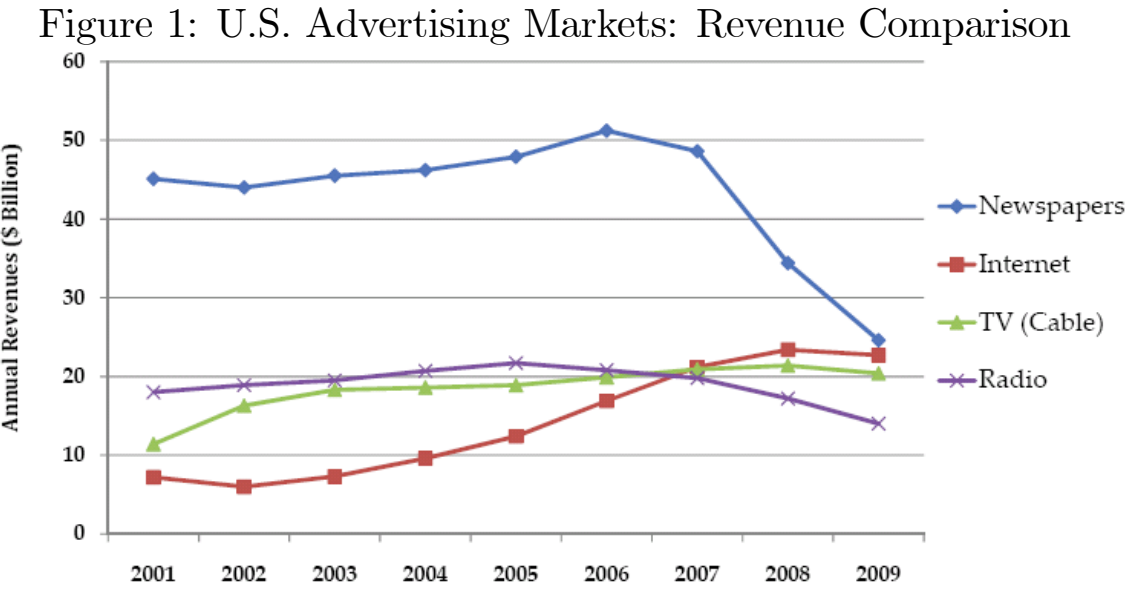

At the same time, through a variety of technological advances, the internet has allowed many advertisers to address a targeted audience beyond the reach of traditional media. In fact, it has been argued that the distinguishing feature of internet advertising is its ability to convey information to a targeted audience. In particular, targeting improves the quality of the match between the consumer and the advertisement message, and enables smaller businesses to access advertising markets from which they were previously excluded. ${ }^{2}$ While this holds for display advertising, it is even more true for sponsored search, where the individual consumer declares her intent or preference directly, by initiating a query.

The objective of this paper is to develop a model of competition between offline (traditional) and online (new) media, in which the distinguishing feature of the online media is the ability to (better) target advertisement messages to their intended audience. We investigate the role of targeting in the determination of (a) the allocation of advertisements across different media, and (b) the equilibrium price for advertising. For this purpose, we first develop a framework to analyze the role of targeting, and then use it to study the interaction between

\footnotetext{
${ }^{1}$ Source: Price Waterhouse Coopers annual reports for the Interactive Advertising Bureau.

${ }^{2}$ Anderson (2006) refers to this phenomenon as the "long tail of advertising."
} 
offline and online advertising.

We present a model in which advertising creates awareness for a product. We consider an economy with a continuum of buyers and a continuum of products, each sold by a different firm. Each product has a potential market size which describes the mass of consumers who are contemplating to purchase it. Each consumer is contemplating only one of the available products, and the role of the advertisement is to generate a match between product and consumer. The placement of an advertisement constitutes a message from the advertiser to a group of consumers. If the message happens to be received by a consumer with interest in the advertiser's product, then the potential customer turns into an actual customer and a sale is realized. A message received by a customer who is not in the market for the product in question is irretrievably lost and generates no tangible benefit for the advertiser. At the same time, a potential customer might be reached by multiple and hence redundant messages from the same advertiser. Consequently, the probability that a potential customer is turned into an actual customer is an increasing but concave function of the number of messages sent.

We begin the analysis with a single advertising market in which all consumers are present and can be reached by any advertiser. It is useful to think of the single advertising market as a national outlet, such as the nationwide newspapers or the major television networks. We show that in this market structure only the largest firms, measured by the size of their potential market, purchase any advertising space. We also show that an increase in the concentration of consumers' interests has an initially positive, but eventually negative effect on the equilibrium price of messages.

We introduce the possibility of targeting by introducing a continuum of advertising markets. Each advertising market is characterized by the composition of the audience in terms of preferences over products. While each consumer is only present in one advertising market, the likelihood of her presence in a specific market is correlated with her preference for a product. As each consumer segment becomes more concentrated in fewer advertising markets, the probability of a match between consumers and advertisements increases. In consequence, the social welfare is increasing with the ability of the advertisers to reach their preferred audience. We then investigate the equilibrium advertising prices as targeting improves. While the marginal product of each message is increasing in the targeting ability, thus potentially increasing the price of advertising, a second and more powerful effect appears. As consumers become more concentrated, the competition among different advertisers becomes weaker. In fact, each advertiser focuses his attention on a few important advertising markets and all but disappears from the other advertising markets. Therefore, the price of advertising is declining in the degree of targeting, even though the value of advertising is increasing. The 
number of participating advertisers shows a similar behavior. While improved targeting increases the total number of advertisers participating across all markets - by allowing smaller advertisers to appear - it reduces the number of actively advertising firms in each specific advertising market. The non monotonicity in the price of advertising is also robust to the introduction of IP address tracking technologies, which eliminate the duplication risk. In fact, the tracking technology eliminates the opportunity for market participants to adjust on the intensive margins (for the largest firms to purchase more messages) and as a result, the equilibrium price declines even faster.

In the second part of the paper we introduce competition among different media for the attention of the consumer. Thus, while each consumer is still only interested in one product, she can now receive a message from any advertiser through two different media. A single message received in either one of the media is sufficient to create a sale. The "dual-homing" of the consumer across the two media markets may then lead to duplicative efforts by the advertisers, who therefore view messages in the two competing markets as substitutes. We first describe the advertising allocation when the competitors are both traditional media without any targeting ability. In this case, messages on the two media are perfect substitutes, and the equilibrium prices are equalized. Furthermore, the allocation of messages only depends on the total supply, not on its distribution across media.

The competition among two offline media markets presents a useful benchmark when we next consider competition between an offline and an online market. We analyze the interaction of offline media - such as newspapers or TV - with online media, such as display (banner) and sponsored keyword search advertisements. Display advertisements allow for targeting through superior knowledge of the consumer's preferences (attribute targeting), while both display and sponsored search advertisements allow advertisers to infer the consumer's preferences from her actions (behavioral targeting). As expected, competition lowers the equilibrium revenues of the traditional medium. However, if entry by an online competitor reduces the available advertising space on the traditional media (for example, by reducing the time consumers spend on each channel), then the effect of competition on the equilibrium price of advertising is non-monotonic. As consumers shift their attention from traditional to new (targeted) media, the price on the traditional channels is first decreasing, then increasing. This has differential implications for the revenues of firms with different potential market sizes. In particular, large firms initially benefit from the consumers' increasing exposure to online advertising, but eventually see their profits decline as the opportunities for offline advertising shrink.

This paper is related to several strands in the literature on advertising and media competition. Anderson and Coate (2005) provide the first model of competing broadcasters, with 
exclusive assignment of viewers to stations. Their setup is extended by Ferrando, Gabszewicz, Laussel, and Sonnac (2004), and Ambrus and Reisinger (2006) to the case of non-exclusive assignments. Dukes (2004) analyzes advertising when the broadcasters and the advertisers compete in a Hotelling-like oligopoly. But the role of targeting for the structure of advertising markets has received scant attention in the literature. The most prominent exception is Iyer, Soberman, and Villas-Boas (2005), who analyze the strategic choice of advertising in an imperfectly competitive market with product differentiation. In their model, the consumers are segmented into different audiences that the firms can target with advertising messages. Yet, Iyer, Soberman, and Villas-Boas (2005) are mostly concerned with the equilibrium prices in the product market that result from the competitive advertising strategies. This focus on the equilibrium price for the advertised products, rather than the equilibrium price of advertising per se is also present in the seminal work by Butters (1977), as well as in more recent work by Esteban, Gil, and Hernandez (2001) and de Cornière (2010). In contrast, we take the products' prices and characteristics as given, and focus our attention on the equilibrium prices of the advertising messages themselves. Finally, the work of Anderson and De Palma (2009), Johnson (2010), and Van Zandt (2004) examine the issues of congestion and privacy, and introduce the possibility that consumers pay selective attention to advertising messages.

In this paper, each advertisement generates a match between a product and a potential customer. The present interpretation of advertising as matching products and users is shared with recent papers, such as Athey and Ellison (2011) and Chen and He (2006). Yet, in these contributions, the primary focus is on the welfare implications of position auctions in a search model where consumers are uncertain about the quality of the match. Similarly, several recent papers, Edelman, Ostrovsky, and Schwarz (2007) and Varian (2007) among others, focus on the specific mechanisms used in practice to sell advertising messages online, such as auctions for sponsored links in keyword searches. In contrast, we model the market for advertisements as a competitive market and the allocation of advertising messages is determined by the competitive equilibrium price.

In closely related work, Athey and Gans (2010) analyze the impact of targeting on the supply and price of advertising in a model with local and general outlets. In their model, targeting improves the efficiency of the allocation of messages, and leads to an increase in demand. They observe that as long as advertising space can be freely expanded, the revenue effects of targeting can also be obtained by increasing the supply of (non targeted) messages, yielding an equivalence result. More generally, Athey and Gans (2010) show that supplyside effects mitigate the value of targeting. Finally, Levin and Milgrom (2010) discuss the trade-off between value creation and market thickness in the context of online advertising, and describe several instances of excessive targeting leading to lower revenues for publishers. 
The remainder of the paper is organized as follows. Section 2 introduces the model and describes the targeting technology. Section 3 opens with the equilibrium analysis in a single advertising market. Section 4 investigates the general model with many advertising markets. Section 5 extends the analysis by allowing each consumer to be present in several media markets. Section 6 investigates the competition between offline and online media. The Appendix collects the formal proofs of all propositions in the main body of the text.

\section{Model}

Advertising and Product Markets We consider a model with a continuum of products and a continuum of advertising markets. Each product $x$ is offered by firm $x$ with $x \in[0, \infty)$. Advertising markets are indexed by $a \in[0, \infty)$. There is a continuum of buyers with unit mass. Each buyer is characterized by two dimensions: his location in a specific advertising market $a$, and his preference for one specific product $x$. The population of consumers is jointly distributed across advertising markets $a$ and product markets $x$ according to $S(a, x)$, with a density $s(a, x)$. For brevity of notation, we often denote the density by $s_{a, x}$.

The fraction of consumers interested in product $x$ is given by the marginal distribution, integrating over all advertising markets:

$$
s(x) \triangleq \int_{0}^{\infty} s\left(a^{\prime}, x\right) d a^{\prime} .
$$

The share of consumers interested in product $x$, denoted by $s(x)$, represents the potential market size of firm $x$. We shall use the notion of product $x$ and firm $x$ interchangeably. The variation in potential market size $s(x)$ across firms allows us distinguish between products with a broad and a narrow audience. Similarly, the size of the advertising market $a$ is given by the marginal distribution, integrating over all products $x$ :

$$
s(a) \triangleq \int_{0}^{\infty} s\left(a, x^{\prime}\right) d x^{\prime}
$$

The consumers of a given product $x$ are distributed across advertising markets according to the conditional distribution:

$$
s(a \mid x) \triangleq \frac{s(a, x)}{s(x)} .
$$

We shall represent changes in the targeting ability of advertising markets as changes in conditional (and unconditional) distribution across advertising markets, all the while maintaining the underlying preference of the consumers, that is the distribution $s(x)$ over products. 
A sale of product $x$ occurs if and only if the buyer is interested in the product and receives at least one message from firm $x$. In the terminology of Bagwell (2007), we adopt the complementary view of advertising, in which the message and the suitable recipient are necessary to generate a purchase. Each sale generates a gross revenue of $\$ 1$, constant across all product markets.

The advertising policy of firm $x$ determines the number of messages $m_{a, x}$ it distributes in advertising market $a$. Each message of advertiser $x$ reaches a random consumer in advertising market $a$ with uniform probability. Given the size of the advertising market $s_{a}$ and the message volume $m_{a, x}$, the probability that a given consumer in market $a$ is aware of product $x$ is then a function of the advertising intensity:

$$
f\left(m_{a, x}, s_{a}\right) \triangleq 1-e^{-m_{a, x} / s_{a}} .
$$

We refer to $f\left(m_{a, x}, s_{a}\right)$ as the awareness level for product $x$ in advertising market $a$. The exponential form of the matching probability (3), is a result of the uniform random matching process. In detail, suppose a large number of messages, denoted by $m$, is distributed with uniform probability across a large number of agents, denoted by $s$. Now, the exact probability that a representative agent has received none out of the $m$ messages is given by:

$$
(1-1 / s)^{m}
$$

By the definition of the exponential function, we have that as $m$ and $s$ approach infinity, while holding the ratio $\mathrm{m} / \mathrm{s}$ constant:

$$
\lim _{m, s \rightarrow \infty}(1-s)^{m}=e^{-m / s}
$$

and the complementary probability is given by (3). As the sales volume of firm $x$ depends on the number of messages it sends, the potential market size $s(x)$ of each firm is precisely that, a potential, whereas the realized market size, the volume of sales, depends on the message volume. With this distinction established, for brevity, we shall refer to $s(x)$ as market size, and for the realized market size as sales.

Finally, we consider a fixed supply of messages $M_{a}$ in every advertising market $a$. The supply of advertising messages in each market is given by the total time/attention devoted by consumers to advertisement messages. In consequence, the supply of messages $M_{a}$ is proportional to the size $s_{a}$ of the advertising market, or

$$
M_{a} \triangleq s_{a} \cdot M
$$


where the constant $M>0$ represents the average time that each individual consumer spends on advertising messages. In the case of broadcast media, the constant $M$ corresponds literally to the time spent watching a television channel or listening to a radio station. In the case of print media and display advertising on the internet, the raw number of advertisements placed and/or the size of the advertisement constitute a reasonable proxy for the attracted time/attention of the consumer.

We assume that each advertising market $a$ is populated with a large number of publishers of advertising messages. Since each publisher acts as a price taker, it follows that firms $x$ purchase advertisement messages at a constant unit price $p_{a}$ in each market $a$. The total profits of firm $x$ are then given by:

$$
\pi_{x} \triangleq \int_{0}^{\infty}\left[s_{a, x} f\left(m_{a, x}, s_{a}\right)-p_{a} m_{a, x}\right] d a .
$$

The seminal work of Butters (1977) introduced the matching technology (3) in the economics of advertising. Yet, in Butters (1977), the price of advertising messages is given exogenously and the price of the product is determined in equilibrium. By contrast, in our model, the price of the advertising is determined in equilibrium and the price of the product is given exogenously. In addition, while Butters (1977) considers many sellers with a homogeneous good, we consider many sellers with heterogeneous products. ${ }^{3}$

Exponential Model In order to efficiently capture the role of product market concentration and advertising market targeting, the allocation of buyers across product and advertising markets is assumed to be governed by an exponential distribution. Firms are ranked, without loss of generality, in decreasing order of market size, so $s_{x}$ is decreasing in $x$ :

$$
s_{x} \triangleq \lambda e^{-\lambda x}
$$

The parameter $\lambda \geq 0$ measures the concentration of consumer's interests, and a larger value of $\lambda$ represents a more concentrated product market. We refer to firms with a low index $x$ as "large firms," to denote the share of consumers interested in their product. In turn, the conditional distribution of consumers interested in product $x$ over advertising markets $a$ is

\footnotetext{
${ }^{3}$ In Bergemann and Bonatti (2010), we show that the competitive outcome can already be attained with a small number of publishers when the individual consumer is spending random (and in expectation uniform) amounts of time with each publisher, even if each firm could restrict its quantity of messages.
} 
given by a (truncated) exponential distribution:

$$
\frac{s_{a, x}}{s_{x}} \triangleq\left\{\begin{array}{cl}
\gamma e^{-\gamma(x-a)}, & \text { if } \quad 0<a \leq x \\
0, & \text { if } \quad x<a<\infty
\end{array}\right.
$$

with a mass point at $a=0$ :

$$
\frac{s_{a, x}}{s_{x}} \triangleq e^{-\gamma x}, \text { if } a=0 .
$$

In other words, we model market $a=0$ as a large advertising market, in which all advertisers are potentially interested (as $s_{x, 0}>0$ for all $x$ ), such as the Yahoo! front page, or a national newspaper. ${ }^{4}$ The parameter $\gamma \geq 0$ measures the concentration of the consumers across the advertising markets. A larger value of $\gamma$ represents a heavier concentration of fewer consumer segments in every advertising market. The corresponding unconditional potential shares are given by:

$$
s_{a, x} \triangleq\left\{\begin{array}{cl}
\lambda \gamma e^{-(\lambda+\gamma) x} e^{\gamma a}, & \text { if } 0<a \leq x, \\
0, & \text { if } x<a<\infty,
\end{array}\right.
$$

with a mass point at $a=0$ :

$$
s_{a, x} \triangleq \lambda e^{-(\lambda+\gamma) x} \quad \text { if } a=0
$$

Consequently, the population size in advertising market $a>0$ is given by the integral over the population shares:

$$
s_{a>0} \triangleq \int_{a}^{\infty} \lambda \gamma e^{-(\lambda+\gamma) x} e^{\gamma a} d x=\frac{\gamma \lambda}{\gamma+\lambda} e^{-\lambda a}
$$

The share of consumers active in product market $x$ and located in advertising market $a=0$ is given by the residual probability of the product market segment $x$. As a result, the population size in advertising market $a=0$ is given by the mass point

$$
s_{a=0} \triangleq \int_{0}^{\infty} \lambda e^{-(\lambda+\gamma) x} d x=\frac{\lambda}{\gamma+\lambda}
$$

For $\gamma>0$, the distribution of consumers over product and advertising markets has a triangular structure. The consumers who are interested in product $x$ are present in all advertising markets $a \leq x$, but are not present in the advertising markets $a>x$.

The distribution of consumers across a one-dimensional product space and a one-dimensional advertising space has a natural interpretation in terms of specialization of preferences and

\footnotetext{
${ }^{4}$ The introduction of a mass point in the conditional distributions $s_{a, x} / s_{x}$ at $a=0$ does not affect the equilibrium properties on market $a$ relative to all other markets $a>0$ as the exponential distribution maintains the relative market shares.
} 
audiences. In this interpretation, a product with a larger index $x$ represents a more specialized product with a smaller population of interested consumers. Correspondingly, an advertising market with a larger index $a$ represents an outlet with a narrower audience. To give a precise example, consider the market for bicycles. Here, products naturally range from mass-produced comfort bikes, to quality-produced fitness bikes, to high-end racing bikes with successively smaller potential shares. Similarly, there is a natural range of advertising markets, from daily newspapers with a large audience, to monthly magazine with well-defined audience such as "Sports Illustrated," to narrowly focused publications, such as "Velonews". Now, the triangular structure of the joint distribution implies that the consumer with an interest in racing bikes may read either one of the publications, but that a consumer with interest in fitness bikes does not read "Velonews," and by extension that a consumer with an interest in comfort bikes does not read "Velonews" nor "Sports Illustrated". In other words, the triangular structure represents a positive but less than perfect correlation of the preference and the audience characteristics of a consumer.

The triangular structure, namely that a consumer with index $x$ is distributed across advertising markets with a smaller index $a$, or $a \leq x$, has some specific implications for the joint distribution of consumers and advertising outlets. In particular, the consumers of larger advertisers are distributed over a smaller number of media outlets, and advertisers of similar size display a greater correlation of consumers and mediate outlets. This implications largely follow from the two-dimensional parametrization of consumers and media outlets. While they are not essential for the qualitative character of our results, they allow us to represent targeting and consumer concentration in terms of the parameters of the exponential distributions, namely $\gamma$ and $\lambda$, respectively.

As we vary the targeting measure $\gamma$ from 0 to $\infty$, we change the distribution and the concentration in each advertising market. The limit values of $\gamma$, namely $\gamma=0$ and $\gamma=$ $\infty$, represent two special market structures. If $\gamma=0$, then all consumers are present in advertising market 0 and hence there is a single advertising market. If, on the other hand, $\gamma \rightarrow \infty$, then all consumers of product $x$ are present in advertising market $x$, and hence we have advertising markets with perfect targeting. More generally, as we increase $\gamma$, an increasing fraction of consumers of product $x$ move away from the large advertising markets (near $a=0$ ) to the smaller advertising markets (near $a=x$ ). Figure 2 illustrates the cross section, represented by the conditional distribution $s_{a, x} / s_{x}$, of how the consumer segments of two different products are distributed across the advertising markets (for a low and high degree of targeting in the left and right panel respectively). The mass points indicate the number of consumers interested in each product that are present in advertising market 0 .

An increase in the degree of targeting also affects the composition of each advertising 
Figure 2: Conditional Distribution of Consumers across Advertising Markets
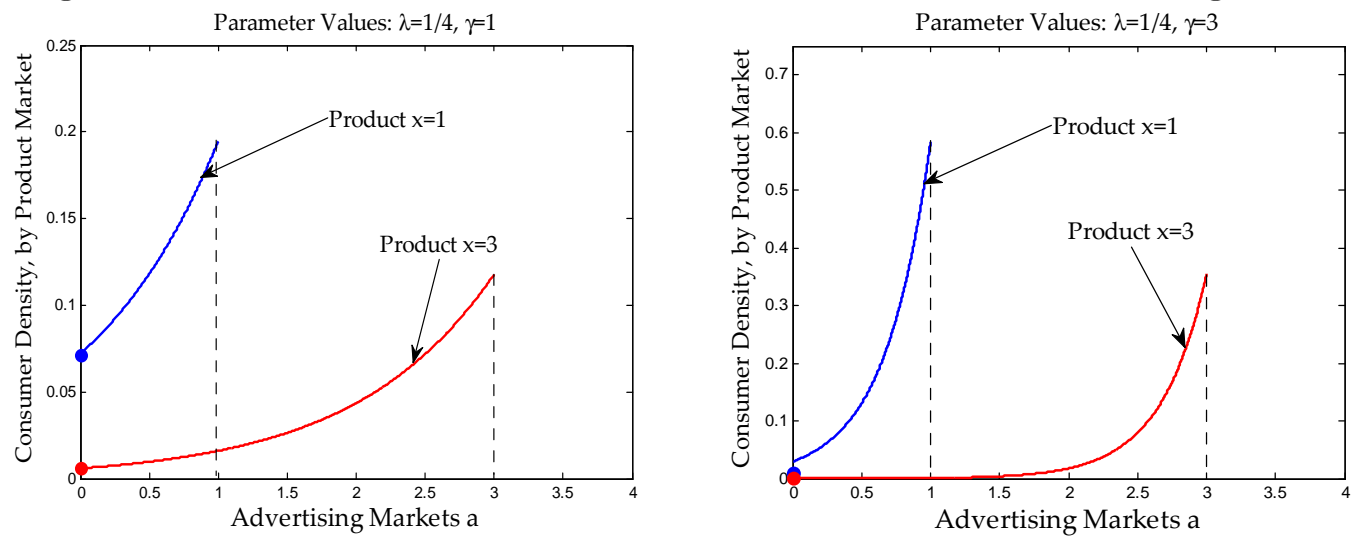

market. In particular, in every market $a$, the naturally targeted product $x=a$ has a relatively larger market size. Figure 3 shows the composition of two different advertising markets, represented by the conditional distribution of consumers' interests $s_{a, x} / s_{a}$, for a low and high degree of targeting, respectively.

Figure 3: Conditional Distribution of Consumers across Product Markets
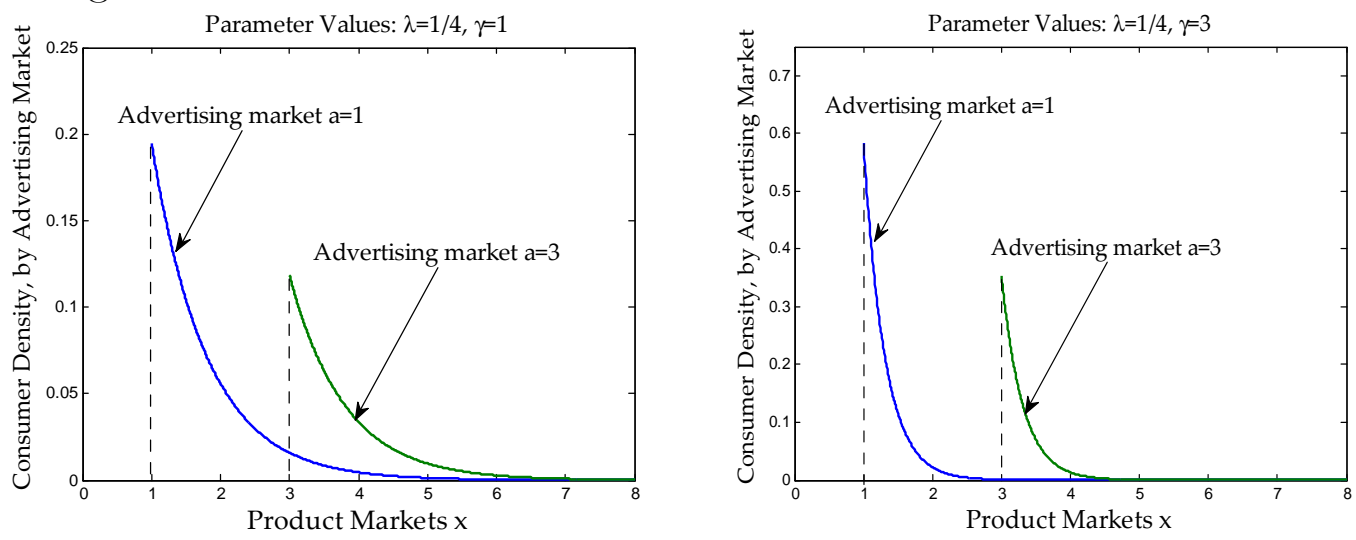

\section{Single Advertising Market}

We begin the equilibrium analysis with the benchmark case in which all consumers are present in a single advertising market. In terms of the distribution of the consumers over the advertising markets, this corresponds to setting $\gamma=0$. Each firm $x$ can now potentially reach all its consumers by placing messages in the single advertising market $a=0$. Consequently, in this section we drop the subscript $a$ in the notation without loss of generality. The objective of each firm $x$ is to maximize the profit given the unit price for advertising $p$. The profit $\pi_{x}$ is given by: 


$$
\pi_{x}=\max _{m_{x}}\left[s_{x} f\left(m_{x}\right)-p m_{x}\right] .
$$

An advertising policy $m_{x}$ generates a gross revenue $s_{x} \cdot f\left(m_{x}\right)$. The information technology $f\left(m_{x}\right)$, given by $(3)$, determines the probability that a representative consumer is aware of product $x$, and $s_{x}$ is the market size of product $x$. The cost of an advertising policy $m_{x}$ is given by $p \cdot m_{x}$. The demand for messages by firm $x$, as determined by the first order conditions, is given by:

$$
m_{x}=\left\{\begin{array}{lll}
\ln \left(s_{x} / p\right) & \text { if } & s_{x} \geq p \\
0 & \text { if } & s_{x}<p .
\end{array}\right.
$$

It is an implication of the above optimality conditions that firms with a larger market size $s_{x}$ choose to send more messages to the consumers. In consequence, at the equilibrium price, the firms with the largest market size choose to advertise. Let $[0, X]$ be the set of participating firms, where $X$ is the marginal firm, and let $M$ be the total supply of messages. The equilibrium price $p$ for messages is then determined by the market clearing condition:

$$
\int_{0}^{X} m_{x} d x=M
$$

Using the optimal demand of firm $x$ and the distribution of market sizes (5), we obtain

$$
\int_{0}^{X}(\ln (\lambda / p)-\lambda x) d x=M .
$$

The equilibrium price and participation are determined by imposing $m_{X}=0$ and the market clearing condition in (9). The competitive equilibrium is characterized by $\left(p^{*}, X^{*}\right)$ with:

$$
\begin{aligned}
p^{*} & =\lambda e^{-\sqrt{2 \lambda M}} \\
X^{*} & =\sqrt{2 M / \lambda}
\end{aligned}
$$

By inserting these formulas into the demand functions of the advertisers, we obtain the competitive equilibrium allocation of messages for a single advertising market with a given capacity $M$ :

$$
m_{x}^{*}=\left\{\begin{array}{cl}
\sqrt{2 \lambda M}-\lambda x, & \text { if } x \leq X^{*}, \\
0, & \text { if } x>X^{*} .
\end{array}\right.
$$

Thus, in the competitive equilibrium the $X^{*}$ largest firms enter the advertising market and the remaining smaller firms stay out of the advertising market. With the exponential distribution of consumers across products, the number of messages sent by an active firm is linear in its rank $x$ in the market. 
We note that in the current environment, the advertising firms face only a pecuniary, or indirect, congestion effect, as messages sent by competing firms do not directly reduce the effectiveness of an advertising campaign. Rather, as other firms demand a larger number of messages, the market clearing price is driven upwards, reducing the demands of each firm $x$. In consequence, the competitive equilibrium implements the socially efficient allocation of advertisement messages (given $\lambda$ ). An easy way to see this is that with a uniform unit price of messages, the marginal returns to the messages bought by different firms are equalized. A natural question is how does the social value of advertising depend on the concentration of the product market. Consider holding the allocation $m_{x}^{*}$ fixed, and increasing $\lambda$. Now the total number of consumers interested in the advertising firms has increased, and thus fewer messages are wasted and more matches are formed. At the new equilibrium (and socially optimal allocation), welfare will be even higher, as the allocation adjusts in favor of the firms with a larger market size.

\section{Proposition 1 (Single Market, Efficiency)}

The social value of advertising is increasing in $\lambda$.

We next determine how the equilibrium allocation depends on the primitives of the advertising market, namely $\lambda$ and $M$.

\section{Proposition 2 (Single Market, Comparative Statics)}

1. The equilibrium demand of messages $m_{x}^{*}$ is increasing in $\lambda$ iff $x \leq X^{*} / 2$.

2. The number of advertising firms $X^{*}$ is increasing in $M$ and decreasing in $\lambda$.

3. The equilibrium price $p^{*}$ is decreasing in $M$ for all $\lambda$.

4. The equilibrium price $p^{*}$ is increasing in $\lambda$ iff $\lambda<2 / M$.

5. The price per consumer reached is increasing in $x$. It is decreasing in $\lambda$ for $x \leq X^{*} / 2$.

The equilibrium price responds to the concentration measure $\lambda$ in a subtle way. If the product market is diffuse, an increase in the concentration increases the market size (and hence the returns from advertising) of all the active firms. This drives up market demand and causes the equilibrium price to increase. Conversely, if the concentration in the product market is already large, then a further increase in the concentration weakens the marginal firm's willingness to pay for advertising. In other words, the demand of the inframarginal firms (whose market size increases) has a positive effect on the price, which is contrasted by the falling demand of the smaller, marginal firms. But as the market size of the large firms 
is already substantial, the increase in their demand is not sufficient to pick up the decrease in demand coming from the marginal firms, and consequently the equilibrium price falls. The additional demand of the large firms is weak because of decreasing marginal returns: an increase in the already large advertising volume leads to many more redundant messages, which generate few additional sales. Figure 4 shows the market demand and supply for different values of the concentration measure $\lambda$.

Figure 4: Equilibrium Demand for Different Concentration Measures

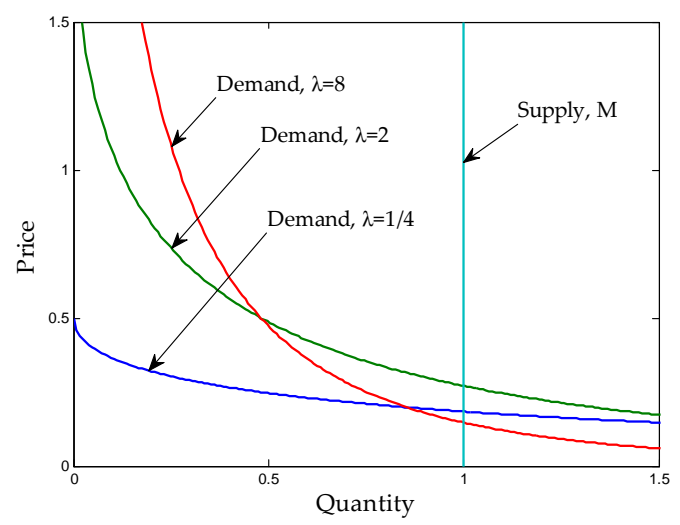

We can view the dichotomy in the comparative statics as driven by the determination of the marginal demand for advertising. For high enough $\lambda$, the source of the marginal demand is the marginal firm, and the price goes down with an increase in $\lambda$. But for low values of $\lambda$, the marginal demand is driven by the inframarginal firms, and then the advertising price is increasing with $\lambda$. The non-monotonic behavior of prices is not specific to the exponential distribution of firms' market sizes, but rather it is a general consequence of the natural tension between competition and concentration.

We note that the non-monotonic behavior of prices is caused by the rotation in the market demand curve shown in Figure 4. The rotation of the demand curve here is related to, but distinct from, the rotation of the distribution of consumer valuations, as analyzed in Johnson and Myatt (2006). In our model, a rotation of the density of consumer tastes induces a rotation in the firms' demand function for advertising, and therefore has a similar effect on the market demand curve as in Johnson and Myatt (2006). In particular, as more consumers are interested in mass products ( $\lambda$ increases), the corresponding larger firms purchase more messages. This shift partially balances the decline in the willingness to pay of the marginal firm, so that the eventual decline in price is rather slow. The effect of a change in the distribution of consumers is therefore mitigated by the firms' adjustment along the intensive margin.

In general terms, the present comparative static analysis appears to be a relevant exercise 
in any product market where the supply is fixed (or has otherwise a specific structure). Johnson and Myatt (2006) pose the comparative static analysis in terms of product design, so that the product becomes more valuable to some customers while less valuable to others. Yet, besides the analysis of Johnson and Myatt (2006), the present point of view seems novel to the literature. We should emphasize that we maintain the competitive equilibrium price mechanism throughout the comparative static analysis. Yet, as the consumers become more concentrated around a smaller set of firms, one could conceivably consider alternative price mechanism which would reflect the increase in bargaining power of the large firms. In turn, this may weaken the downward trend in prices as $\lambda$ increases.

It is useful to recast the equilibrium of our model in hedonic terms. In this respect, Proposition 2 shows that larger firms pay a decreasing amount per consumer reached as $\lambda$ increases. This result is driven by the concentration of the equilibrium messages in the hands of a few firms, who make large profits on the inframarginal units. Conversely, the price per consumer reached is increasing in $\lambda$ for firms smaller than the median advertising firm. For these firms, the price per consumer reached increases until it attains a value of one (which is the marginal return to the first message $f^{\prime}(0)$ ). In particular, for all $\lambda$, the marginal firm $X^{*}(\lambda)$ pays a price per consumer reached equal to one.

Relaxing the assumption of perfectly inelastic supply only affects some of the comparative statics result in Proposition 2. For the case of constant supply elasticity $q=M p^{\varepsilon}$, we can show that the equilibrium price retains the same comparative static properties: it is first increasing, then decreasing in $\lambda$. Moreover, as $M$ becomes larger, the equilibrium price will be increasing in $\lambda$ over a larger range. In particular, when the product market is very concentrated, so that the willingness to pay of the marginal firm is low, a more elastic supply reduces the number of active firms in the market. For high values of $\lambda$, it continues to hold that the demand falls off fast enough that the equilibrium price decreases. In particular, as $\lambda$ goes to infinity, both the price and the quantity traded go to zero. However, since an increase in $\lambda$ causes a drop in the quantity sold, the welfare result with respect to an increase in the concentration measure $\lambda$ now becomes ambiguous.

\section{Many Advertising Markets}

We are now in a position to analyze the general model with a continuum of advertising markets. We described the distribution of consumers over different advertising markets by a (truncated) exponential distribution with a positive targeting parameter $\gamma \in(0, \infty)$. The share of consumers in product category $x$ and located in advertising market $a$ is given by (6). The case of a advertising market with zero targeting is described by $\gamma=0$, while the 
case of perfect targeting is described by $\gamma=\infty$.

An important implication of the exponential distribution across advertising and product markets is a certain stationarity in the composition over the consumers across the advertising markets. In particular, the relative shares of the product markets are constant across advertising markets:

$$
\frac{s_{a, x}}{s_{a}}=(\lambda+\gamma) e^{-(\lambda+\gamma)(x-a)}=\frac{s_{a+n, x+n}}{s_{a+n}},
$$

for all $x \geq a$ and all $n \geq 0$. Thus, while the exact composition of each advertising market is changing, the size distribution of the competing advertisers remains constant across advertising markets. This stationarity property allows us to transfer many of the insights of the single advertising market to the world with many advertising markets.

Now we consider the optimization problem of firm $x$ in market $a$,

$$
m_{a, x}=\underset{m}{\arg \max }\left[s_{a, x}\left(1-e^{-m / s_{a}}\right)-p_{a} m\right] .
$$

The demand function of firm $x$ in market $a$ is then by:

$$
m_{a, x}=s_{a} \ln \left(s_{a, x} / p_{a} s_{a}\right)
$$

The equilibrium in each market $a$ is determined through the demand functions (13), the marginal firm $X_{a}$ :

$$
s_{a, X_{a}} / s_{a}=p_{a}
$$

and the market clearing condition:

$$
\int_{a}^{X_{a}} m_{a, x} d x=s_{a} M
$$

We now characterize the equilibrium prices $p_{a}^{*}$, the number of active firms $X_{a}^{*}-a$, and the allocation $m_{a, x}^{*}$ of messages. The price and the number of active firms are stationary in the index $a$ of the advertising market, that is:

$$
\begin{aligned}
p_{a}^{*} & =(\gamma+\lambda) e^{-\sqrt{2 M(\gamma+\lambda)}}, \\
X_{a}^{*}-a & =\sqrt{2 M /(\gamma+\lambda)},
\end{aligned}
$$

for all $a \geq 0$. Observe that the stationarity of the equilibrium prices implies that the marginal utility of an additional message is equalized across markets. We also know that the competitive equilibrium allocation of the advertising space $M_{a}$ in each market is efficient. Therefore, the efficient allocation of a fixed advertising space $M$ is proportional to the size of 
the advertising market: $M_{a}=s_{a} \cdot M$. In other words, if the social planner had the opportunity to rearrange the supply of messages across markets, she would not find it optimal to do so. Finally, the allocation of messages is given by

$$
m_{a, x}^{*}=\left\{\begin{array}{cc}
\gamma \lambda e^{-\lambda a}(\sqrt{2 M /(\gamma+\lambda)}-(x-a)), & \text { if } a>0 \\
\lambda(\sqrt{2 M /(\gamma+\lambda)}-x), & \text { if } a=0
\end{array}\right.
$$

Clearly, the larger firms $x \geq a$ receive a higher fraction of the message supply. If in particular we consider firm $x=a$, then the number of messages it receives is also increasing in the targeting ability. The comparative statics results with respect to the concentration measure $\lambda$ and message volume $M$ do not differ qualitatively from the case of a single advertising market. More importantly, the effect of targeting ability $\gamma$ and product market concentration $\lambda$ on the equilibrium allocation is remarkably similar. In particular, prices are increasing in $\lambda$ if and only if both the concentration and the targeting parameter are low enough. We can now turn to the comparative statics with respect to $\gamma$, where a higher $\gamma$ means more precise targeting.

\section{Proposition 3 (Social Value of Targeting)}

The social value of advertising is strictly increasing in the targeting ability $\gamma$.

To understand the implications of targeting on social welfare, consider the relative size of consumer segment $x$ in advertising market $a=x$ :

$$
\frac{s_{x, x}}{s_{a=x}}=\gamma+\lambda
$$

We observe that better targeting increases the value that firm $x$ assigns to a message in the advertising market $a=x$. Now let us consider holding the allocation of messages $m_{a, x}$ constant, and increasing the degree of targeting $\gamma$. The volume of matched consumers and firms is increasing because of the shift in the relative sizes of the advertising markets. Since we know that the competitive allocation of messages is Pareto efficient, the equilibrium (for the new $\gamma$ ) has unambiguously improved the social value of advertising.

We now look at the cross-sectional implications of targeting. We find that all smaller firms $x$, namely those that do not participate in the mass advertising market $a=0$, given by:

$$
x>\bar{X}(\gamma) \triangleq \sqrt{2 M /(\lambda+\gamma)}
$$

unambiguously benefit from increased targeting. Similarly, the largest firms benefit from an improved targeting. In contrast, the number of matches achieved by medium sized firms 
(those participating on market $a=0$ but purchasing a small number of messages) is initially decreasing, and only eventually increasing in the level of targeting.

\section{Proposition 4 (Matching across Firms)}

1. The total number of matches generated by firms $x \geq \bar{X}(\gamma)$ is increasing in $\gamma$.

2. There exists a threshold $\bar{x}(\gamma) \in[0, \bar{X}(\gamma)]$ such that the number of matches generated by firm $x$ is increasing in $\gamma$ for $x \leq \bar{x}(\gamma)$ and decreasing for $x>\bar{x}(\gamma)$.

3. If $M \gamma \geq 1+\sqrt{2 M \lambda+1}$, then the number of matches is increasing in $\gamma$ for all firms.

To obtain some intuition for this result, notice that the firms $x \leq \bar{X}(\gamma)$ (the large firms that participate in advertising market $a=0$ ), can find their consumers concentrated in a small number of markets, for all levels of targeting. An increase in the targeting ability improves their chances of achieving a match, but these firms keep a strong presence on the largest advertising market $a=0$. Small firms are not active on market $a=0$. At the same time, the number of consumers present on their "natural" advertising markets $a \approx x$ is increasing in $\gamma$. These firms can now reach a larger fraction of their potential customers. However, the medium sized-firms are hurt by the decrease in the consumer population in market $a=0$, while the increase in the size of their natural markets $a \approx x$ is not sufficient, for low $\gamma$, to compensate for these losses.

We now turn to the effect of targeting on the allocation of messages in each market, and on the equilibrium price of advertising. The revenues from advertising on each market $a$ are defined as $R_{a}^{*} \triangleq s_{a} p_{a}^{*}$.

\section{Proposition 5 (Advertising Demand and Targeting)}

1. The number of messages per capita $m_{a, x}^{*} / s_{a}$ is increasing in $\gamma$ for $x \leq\left(a+X_{a}^{*}\right) / 2$, and decreasing in $\gamma$ otherwise.

2. The number of participating firms $X_{a}^{*}-a$ is decreasing in $\gamma$.

3. The equilibrium price $p_{a}^{*}$ is increasing in $\gamma$ if and only if $\lambda+\gamma<2 / M$.

4. The equilibrium revenue $R_{0}^{*}$ is decreasing in $\gamma$. The revenues $R_{a>0}^{*}$ are increasing in $\gamma$ if and only if $\gamma<(1+\sqrt{1+2 M \lambda}) / M$.

The equilibrium number of messages $m_{a, x}^{*}$ is increasing in $\gamma$ for the participating firms larger than the median active firm. Furthermore, more precise targeting implies a lower number of active firms. Notice that the relationship between targeting ability and equilibrium 
price is generally hump-shaped. However, if either $M$ or $\lambda$ are large, then $p_{a}^{*}$ is decreasing in $\gamma$ for all values of $\gamma$. In other words, despite the increased social value of advertising, the equilibrium price of advertising is decreasing in the targeting ability over a large range of parameter values. In terms of revenues, we can infer from (7) and (8) that an increase in $\gamma$ leads to an increase in the size of markets $a>0$ and to a decrease in the size of market 0 . Since prices are constant, revenues in market 0 are decreasing in $\gamma$. Finally, targeting has the same qualitative effect on the equilibrium revenues in all markets $a>0$.

We now come back to the similar effects of product market concentration and targeting. In particular, as with concentrated product markets, an increase in targeting $\gamma$ reduces the demand of the marginal firm on each advertising market $a$. At the same time, better targeting increases the demand of the inframarginal firms. The underlying tension is the one between identifying a consumer segment precisely, and finding many advertisers who are interested in it.

Robustness We should point out that the exponential distributions over advertising and product markets provide particularly tractable expressions. The insights about the nonmonotonic behavior of the equilibrium price of advertising extend to more general production and distribution functions. In the working paper version, Bergemann and Bonatti (2010), we present a set of sufficient conditions for the comparative static to remain true beyond the exponential model presented here. A prominent example that falls under these conditions is the case of Pareto-distributed consumers over product and advertising markets. The key difference with the exponential distribution lies in the fat tails (and hence decreasing hazard rate) of the Pareto distribution. In the product markets, this means two niche (high $x$ ) products have more similar market sizes, compared to two mass (low $x$ ) products. Analogously, consumers in smaller advertising markets are relatively more dispersed than in larger advertising markets. It follows that, in small advertising markets, the marginal and inframarginal firms have more similar message demands under the Pareto than under the exponential distribution. The number of active firms in each advertising market is then no longer a constant, but rather it is increasing in $a$. In consequence, the willingness to pay of the marginal firm is decreasing in $a$, and therefore so are the equilibrium prices $p_{a}$.

Behavioral Tracking The present random matching of messages and consumers implies a certain level of duplication risk. It is then of some interest to see how the analysis would be affected if the publishers could avoid the duplication risk. ${ }^{5}$ Suppose therefore that the

\footnotetext{
${ }^{5}$ We would like to thank the Co-Editor, Mark Armstrong, for his suggestion to analyze the linear environment without duplication risk.
} 
publisher had access to a technology, such as IP address tracking, that would allow each publisher to avoid the duplication risk, by keeping track of which consumers have been exposed to which messages. In such an environment, each advertiser's marginal utility of messages is no longer decreasing. In particular, in this "linear" advertising environment, if the price is sufficiently low, the advertisers purchase messages that reach the entire consumer population on a given advertising market. In other words, all participating firms buy an impression of one message per consumer. We maintain the proportionality assumption between the number of consumers in an advertising market and the total supply of messages: $M_{a}=s_{a} \cdot M$. In this advertising environment with behavioral tracking, the equilibrium price in advertising market $a$ would again be given by, as before in (14):

$$
p_{a}=\frac{s_{a, X_{a}^{*}}}{s_{a}}
$$

where $X_{a}^{*}=a+M$ is the marginal firm on market $a$. Importantly, all participating firms $x \in\left[a, X_{a}^{*}\right]$ would now buy the same number of messages. Because of the lack of adjustment along the intensive margin, the identity of the marginal firm does not change with the targeting technology. In other words, as $\gamma$ increases, in this linear model there would be no reallocation of messages towards the larger firms. The resulting equilibrium price would be given by:

$$
p_{a}=(\lambda+\gamma) e^{-(\lambda+\gamma) M}
$$

which decreases faster in $\gamma$ than the equilibrium price given in (15). We can therefore conclude that tracking technology does not necessarily help avoid the decline in advertising prices as the targeting ability improves. On the contrary, since the allocation of messages is held fixed, the equilibrium price perfectly tracks the size of the marginal firm's potential market, and hence decreases much faster than in the absence of a tracking technology.

Empirical Evidence Our result on non-monotonic prices finds supporting evidence in the empirical literature. Chandra and Kaiser (2010) considers the advertising market for magazines where advertisers choose among outlets based on the average reader's characteristics. In this market with a low degree of targeting as measured by standard concentration indices, Chandra and Kaiser (2010) exploit data on both readers' characteristics and magazines' advertising prices to estimate the value of targeting. Consistent with the results in Proposition 5 , they find a positive and significant relationship between the homogeneity in a magazine's subscribers' characteristics and the advertising prices charged by its publisher.

In contrast, Rutz and Bucklin (2011) analyze the market for Internet search advertising. By construction, this market allows for very precisely targeted advertisements, as each user's 
keyword search reveals information about her preferences. Rutz and Bucklin (2011) compare branded keyword searches with broader keyword searches, e.g. "Hilton Hotel L.A." vs "Hotel L.A." They find that the measures of consumer response (e.g. click-through rate and conversion rate) to be much higher for branded keywords, which we interpret as very narrowly targeted, compared to the broader, generic keywords. At the same time, the price paid by advertisers for sponsored links on generic keyword search pages is considerably higher, supporting the downward trend in prices for highly targeted ads, as established in Proposition 5. Here we should add the caveat that the measured consumer response may overestimate the true marginal responsiveness of the consumer as the presence of algorithmic (organic) search results would suggest that a consumer searching for "Hilton Hotel L.A." may find the desired Hilton Hotel even if Hilton were not to advertise its keyword listing.

\section{Media Competition}

In this section, we deploy our model of targeting to provide insights into the effects of competition between new and established media. For this reason, we shall weaken the single-homing assumption to allow each consumer to be present in multiple markets. A first effect of competition is then to multiply the opportunities for matching an advertiser with a customer.

We initially consider competition between traditional media, i.e. sellers of non-targeted messages, where each medium is described by a single advertising market. For example, this may represent the competition between nation-wide TV broadcasting and nation-wide newspaper publishers, or between different types of TV networks. We initially abstract away from the role of targeting, in order to trace out the implications of (a) the number of consumers present on each market, and (b) the distribution of consumer characteristics in each market. The analysis of competition between traditional advertising markets can shed light on the interaction of new and established (offline and online) media along at least two dimensions.

First, new media are likely to have an initially smaller user base. As a consequence, advertisement messages have a more narrow reach, though a smaller market makes it easier to reach a large fraction of the audience. Our results show that only the largest advertisers buy a positive number of messages in both markets. Furthermore, these firms purchase a constant number of advertising messages in the (new) smaller market. Therefore, media competition allows medium-sized firms to have a relatively larger presence in the new advertising market, compared to the case of a single and established market.

Second, the main feature of a targeted, online advertising market is a higher concentration 
of consumers of a particular product, compared to a traditional market. Therefore, the degree of product market concentration, which we focus on here, plays a similar role to the degree of advertising market targeting of Section 4. In particular, differences in market concentration lead firms to sort into those markets where their messages have a higher probability of forming a match with the desired customer segment.

Competition by Symmetric Offline Media We begin the analysis with a model of competition between two traditional media. The two media $i \in\{1,2\}$ have the same distribution of consumer characteristics $s_{x}$ in their respective advertising markets and compete for advertisers. Let $M_{i}$ denote the exogenous supply of advertising space on each market. This model provides a useful benchmark to understand the effects of different user bases and consumer distributions.

As in our baseline model, the fraction of consumers reached by firm $x$ on media market $i$ is given by

$$
f_{i, x} \triangleq 1-e^{-m_{i, x}} .
$$

The novel feature of media competition is that each firm $x$ views messages displayed in advertising markets 1 and 2 as (perfect) substitutes. We can therefore define the total awareness level generated by firm $x$ as:

$$
f\left(m_{1, x}, m_{2, x}\right) \triangleq f_{1, x}+f_{2, x}-f_{1, x} f_{2, x}=1-e^{-m_{1, x}-m_{2, x}} .
$$

As each consumer is dual-homing, there is a loss in the frequency of productive matches generated by messages in market 1 because the consumer may have received a duplicate message in market 2 (and conversely). Each firm $x$ maximizes its profit function $\pi_{x}$ :

$$
\pi_{x} \triangleq s_{x} f\left(m_{1, x}, m_{2, x}\right)-\sum_{i \in\{1,2\}} p_{i} m_{i, x} .
$$

It follows that the demand function of firm $x$ in market $i$ is given by

$$
m_{i, x}=\ln \left(\lambda / p_{i}\right)-m_{-i, x}-\lambda x .
$$

This expression differs from the demand function in a single advertising market only because of the perfect substitutability of messages across markets. Intuitively, each firm advertises in medium $i$ until the critical level at which the value of advertising in $i$ falls below $p_{i}$. This level depends on the amount of advertising in the other market. We denote by $m_{x} \triangleq \Sigma_{i} m_{i, x}$ the total number of messages demanded by firm $x$, and we describe the equilibrium allocation in the following proposition. 


\section{Proposition 6 (Offline Media)}

The equilibrium with two competing offline media is given by:

$$
p_{1}^{*}=p_{2}^{*}=\lambda e^{-\sqrt{2 \lambda\left(M_{1}+M_{2}\right)}}
$$

and:

$$
m_{x}^{*}=\sqrt{2 \lambda\left(M_{1}+M_{2}\right)}-\lambda x, \text { for } x \leq X^{*}=\sqrt{2\left(M_{1}+M_{2}\right) / \lambda} .
$$

Since the messages on the two markets are perfect substitutes, it is intuitive that the equilibrium prices must also be identical. The number of active firms $X^{*}$ in equilibrium reflects the increase in the total supply of messages $\left(M_{1}+M_{2}\right)$, but it is otherwise analogous to the case of a single advertising market.

In this symmetric model, the equilibrium allocation of messages is not characterized in terms of each $m_{i, x}$. This is because perfect substitutability of messages across the two media leads to an indeterminacy in the division of message purchases across the two media. In particular, both media specialization - in which each firm $x \leq X^{*}$ buys messages exclusively on one market - and proportional representation of advertisers on each market, may occur in equilibrium. The equilibrium revenues of market $i$ are non-monotonic in the supply level $M_{i}$ and decreasing in $M_{-i}$. Therefore, if we considered advertising space $M_{i}$ as a strategic variable - such as a capacity choice - then market interaction would be analogous to quantity competition between the two media.

Media Markets of Different Size We now turn to the effects of introducing a new advertising medium with a smaller user base, which is visited only by a subset of the consumers. To capture this asymmetry between the new and the established medium in a simple way, let the number of consumers present on (new) market 2 be given by $\delta \leq 1$. Furthermore, all consumers who visit the new medium 2 also visit the established medium 1 . For example, one may think of the early days of online advertising, or more recently the emergence of new social online advertising, as on Facebook or Twitter.

We normalize the supply of messages per capita to $M_{i}$ in each market $i$. Since each firm $x$ can reach a subset of its customers on the new market, the profit function is given by

$$
\pi_{x}=\lambda e^{-\lambda x}\left(\left(1-e^{-m_{1, x}}\right)+e^{-m_{1, x}} \delta\left(1-e^{-m_{2, x} / \delta}\right)\right)-\sum_{i \in\{1,2\}} p_{i} m_{i, x} .
$$

Whenever firm $x$ buys a positive number of messages on both media, the first order conditions 
imply the following demand functions:

$$
m_{1, x}=\ln \frac{\lambda(1-\delta)}{p_{1}-\delta p_{2}}-\lambda x, \quad m_{2, x}=\delta \ln \frac{p_{1}-\delta p_{2}}{p_{2}(1-\delta)}
$$

In particular, for those firms buying on both markets, $m_{1, x}$ is decreasing in $x$, while $m_{2, x}$ is constant in $x$. In other words, the largest firms enter the new market with a constant number of messages. Intuitively, larger firms stand more to lose by shifting messages to the new market and reaching fewer potential customers. More formally, suppose (as is the case) that larger firms buy a larger number of messages on the established market. Given the substitutability of messages across markets, this increases the demand by smaller firms in the new market. In equilibrium, this effect exactly offsets the differences in demand due to firm size, and the resulting allocation of messages on market 2 is flat for all dual-homing firms. Compared to the single market case, the new advertising market is then characterized by a strong presence of "medium-size" firms, and by a longer tail of smaller firms.

In order to complete the description of the equilibrium allocation, we identify two thresholds, $X$ and $Z$, such that firms $x \in[0, X]$ buy messages on both markets, while firms $x \in[X, Z]$ only buy a positive number of messages on market 2 .

\section{Proposition 7 (New Advertising Medium)}

1. The equilibrium allocation of messages in the established market 1 is

$$
m_{1, x}^{*}=\sqrt{2 \lambda M_{1}}-\lambda x, \quad \text { for } x \leq \sqrt{2 M_{1} / \lambda} .
$$

2. The equilibrium allocation of messages in the new market 2 is given by

$$
m_{2, x}^{*}=\left\{\begin{array}{ccc}
\delta\left(\sqrt{2\left(M_{1}+M_{2}\right) \lambda}-\sqrt{2 M_{1} \lambda}\right), & \text { for } & x \leq \sqrt{2 M_{1} / \lambda}, \\
\delta\left(\sqrt{2\left(M_{1}+M_{2}\right) \lambda}-\lambda x\right), & \text { for } & \sqrt{2 M_{1} / \lambda}<x \leq \sqrt{2\left(M_{1}+M_{2}\right) / \lambda}
\end{array}\right.
$$

3. The equilibrium prices are given by

$$
\begin{aligned}
& p_{1}^{*}=\delta \lambda e^{-\sqrt{2\left(M_{1}+M_{2}\right) \lambda}}+(1-\delta) \lambda e^{-\sqrt{2 M_{1} \lambda}}, \\
& p_{2}^{*}=\lambda e^{-\sqrt{2\left(M_{1}+M_{2}\right) \lambda}} .
\end{aligned}
$$

Figure 5 illustrates the allocation for $M_{1}=M_{2}=1, \lambda=2$, and several values of $\delta$. When $\delta=1$, we return to the case of symmetric advertising markets, and the specific allocation displayed below is just one of the possible equilibrium allocations. The displayed allocation for $\delta=1$ is however the unique limit for the equilibrium allocations as $\delta \rightarrow 1$. 
Figure 5: Equilibrium Demand for Different Market Sizes

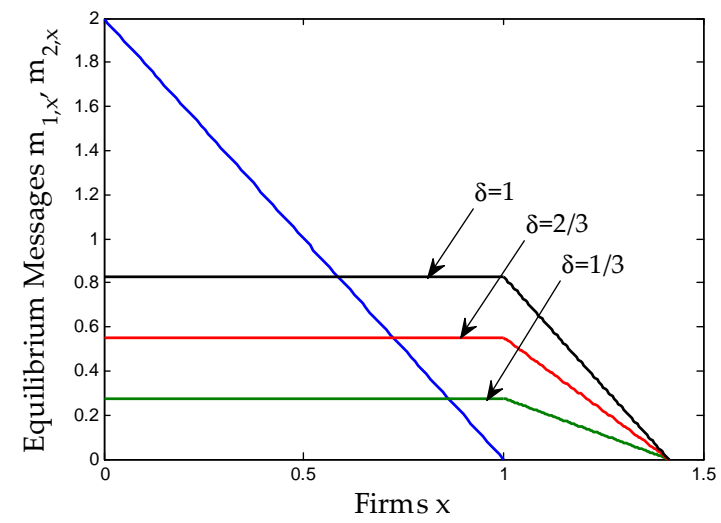

Proposition 7 shows that the number of active firms in market 1 is determined by the single market threshold, when supply is equal to $M_{1}$. The total number of active firms is instead determined by the symmetric competition threshold, when supply is equal to $M_{1}+M_{2}$. Finally, the equilibrium price on the larger market $p_{1}$ is decreasing in the size of the smaller market $\delta$, while the price on the smaller market $p_{2}$ is independent of $\delta$. Both results can be traced back to changes in the supply of messages in the new market. Indeed as $\delta$ increases, demand by the larger advertisers also increases. This would drive the price up and reduce the number of active firms, but this effect is offset by a proportional increase in supply.

Media Markets with Different Distributions As we saw in Section 4, the key advantage of targeted advertising markets is that fewer firms deliver messages to a more concentrated consumer population. We now shift our attention to the role of the distribution of consumer characteristics for the competition between different media markets.

We consider two advertising markets, $i \in\{1,2\}$ and let the distribution of consumers in market $i$ be given by $s_{i, x} \triangleq \lambda_{i} \exp \left(-\lambda_{i} x\right)$. We assume that the advertising market 1 has a more concentrated distribution over consumer characteristics than advertising market 2 , or $\lambda_{1}>\lambda_{2}$. As the distribution of consumers differs across advertising markets, it follows that not all consumer are dual-homing. In particular, if a firm $x$ has a larger presence in market 1 , then all its potential customers are present in market 1, but only a subset of them is present in market 2. Given that $\lambda_{1}>\lambda_{2}$, this is the case for the larger firms, for which $s_{1, x}>s_{2, x}$. The converse holds for the smaller firms, which have more consumers in market 2. The profit function of a large firm $x$ (for which $s_{1, x}>s_{2, x}$ ) can be written as:

$$
\pi_{x}=s_{1, x} f\left(m_{1, x}\right)+\left(1-f\left(m_{1, x}\right)\right) s_{2, x} f\left(m_{2, x}\right)-\sum_{i \in\{1,2\}} p_{i} m_{i, x} .
$$


Thus, firm $x$ perceives market 2 as a lower-quality substitute, analogous to a market with a smaller user base. Market 1 plays a similar role for smaller firms, for which $s_{1, x}<s_{2, x}$. It follows that larger firms have an incentive to focus on medium 1 and to disregard medium 2. The equilibrium allocation is now characterized by three threshold firms, $X<Y<Z$ :

1. The largest firms $x \in[0, X]$ only buy on market 1 .

2. A set of medium-sized firms $x \in[X, Y]$ buy on both markets in varying proportions. In particular, the demand for messages in market 1 is decreasing in $x$, while the demand on market 2 is increasing in $x$. The total demands are decreasing in $x$.

3. The smaller firms $x \in[Y, Z]$ only buy on market 2 .

In equilibrium, the more concentrated market attracts the largest, most valuable, firms. In particular, large firms advertise exclusively on the more concentrated market, while a subset of medium-sized firms advertise on both, and relatively smaller firms only advertise on the more diffuse market. The cutoff values $X, Y$ and $Z$ solve the market clearing conditions given the demand functions. The equilibrium market shares do not allow for an explicit expression in the case of different concentration levels, and the details of the equilibrium construction are presented in Bergemann and Bonatti (2010). In Figure 6, we show the allocations of messages $m_{1, x}$ and $m_{2, x}$ as a function of $\lambda_{1}$. The remaining parameter values are $\lambda_{2}=1$, and $M_{1}=M_{2}=1$.

Figure 6: Equilibrium Demand for Different Concentration Measures
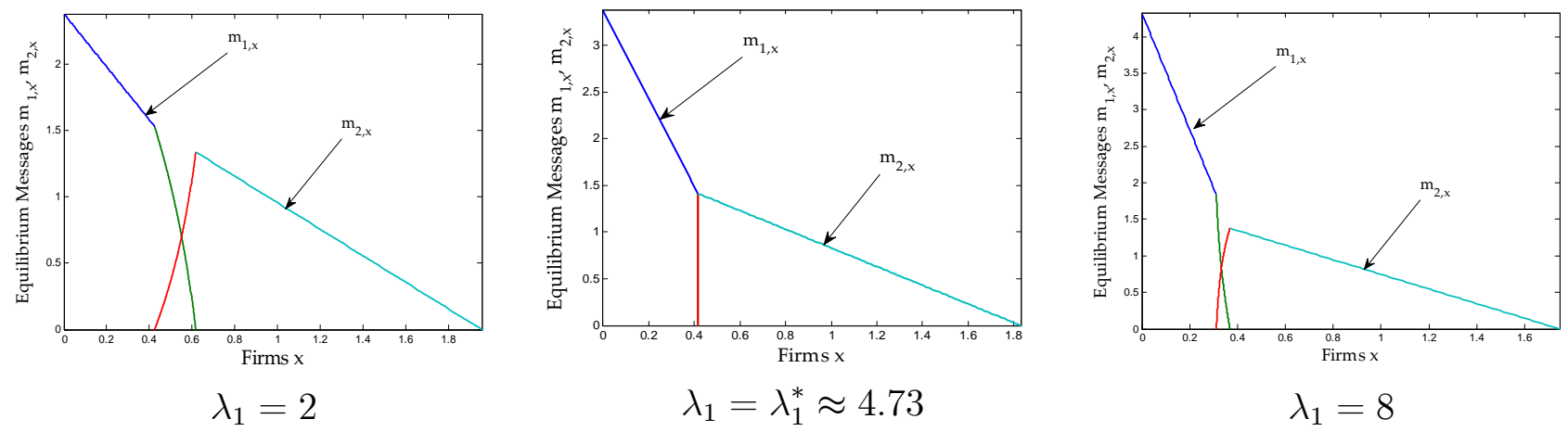

For large differences in the concentration levels $\lambda_{i}$, all dual homing firms $x \in[X, Y]$ satisfy $s_{1, x}<s_{2, x}$, which means they are located to the right of the crossing point of the two density functions. For small differences in the concentration levels, all $x \in[X, Y]$ satisfy $s_{1, x}>s_{2, x}$. For a given choice of the parameters $\left(\lambda_{2}, M_{1}, M_{2}\right)$, the number of dual-homing 
firms $(Y-X)$ is non-monotonic in $\lambda_{1}$, and it is equal to zero for a single value $\lambda_{1}=\lambda_{1}^{*}$. When this is the case, the marginal firm $X=Y$ has an identical market size under both distributions.

The present results provide two kinds of insights into the interaction of online and offline advertising markets. Indeed, we can view each online advertising market as a separate medium with a higher concentration of consumers. With this interpretation, the prediction of the model is that Internet advertising induces the largest, most profitable advertisers to switch away from the offline medium, and to advertise only on the more concentrated online markets.

In this sense, competition by a more concentrated (targeted) market is very different from an (identical) emerging market with a smaller user base. In the former case, the established media lose the most valuable firms, as these firms find a more profitable market where to reach their customers. In the latter case, the established media share the largest buyers with the new media, and actually hold a relatively favorable position (in terms of the allocation of messages purchased by the largest firms).

In an alternative interpretation, we can view market 2 as the newer medium, such as the Internet, with a relatively larger presence of consumers of small (long tail) firms. Competition with a more concentrated (established) market then causes the demand for messages by smaller firms to completely crowd out the demand of larger firms, and to partially offset the demand of medium-size firms. In this sense, online advertising increases the number of firms that have access to messages in equilibrium, and allows for a more significant participation of smaller firms.

\section{Offline vs. Online Media}

The internet has introduced at least two technological innovations in advertising, namely (a) the ability to relate payments and performance (e.g. pay per click), and (b) an improved ability to target advertisement messages to users. We focus on the latter aspect, and in particular on the equilibrium allocation of advertising when both traditional and targeted media are present.

In our model, the targeted markets represent specialized websites, and messages can be thought of as display advertisements. We therefore refer to the traditional medium as "offline," and to the many targeted markets as "online." We then consider a population of dual-homing consumers, who spend a total time of $M_{1}$ on the offline medium, and $M_{2}$ on a single market $a \in \mathbb{R}_{+}$in the online, targeted, medium. More specifically, $s_{a} M_{2}$ denotes the supply of messages on each targeted market. 
Because of the risk of duplication, messages sent online and offline are viewed as substitutes by each firm. This is not the case for messages sent on two different online markets, since each consumer only visits one website (in addition to the offline market). Therefore, if firm $x$ sends a total of $m_{x}$ non-targeted messages and $m_{a, x}$ messages on each online market $a$, its profit function is given by

$$
\pi_{x}=\int_{0}^{x}\left(s_{a, x}\left(1-e^{-m_{x}-m_{a, x} / s_{a}}\right)-p_{a} m_{a, x}\right) d a-p m_{x} .
$$

The analysis of firms' advertising choices between offline and online media is intricate. In general, each firm $x$ will want to advertise on a subset of the online markets $a \leq x$ where its consumers are located (see Figure 2), and some firms will also advertise offline. Both for tractability concerns, and to focus on the revenue implications of competition and targeting, we assume that the online medium allows to perfectly target messages to consumers. We then ask what is the equilibrium unit price of advertisement messages, and how it is affected by each firm's demands offline.

With perfect targeting, each advertising market $a$ is only visited by consumers of product $a$. Since the size of market $a$ is identical to the market size of firm $x=a$, we immediately obtain the allocation and prices online from the individual firms' demands:

$$
\begin{aligned}
& m_{x, x}=\lambda e^{-\lambda x} M_{2}, \\
& p_{a=x}=e^{-M_{2}} e^{-m_{x}} .
\end{aligned}
$$

Equation (18) implies that in equilibrium, given the supply of messages on each market, each firm reaches a constant fraction $1-\exp \left(-M_{2}\right)$ of its customers. ${ }^{6}$ Equation (19) shows that the more firm $x$ advertises offline, the lower the price on the corresponding online market $a=x$. This is again a consequence of the substitutability of messages across media.

We now turn to the message demands offline. Since each firm reaches a constant fraction $1-\exp \left(-M_{2}\right)$ of its customers online, the supply of messages online simply acts as a scaling factor for each firm's demand function offline. Intuitively, each firm now has $s_{x} \exp \left(-M_{2}\right)$ potential customers offline. The equilibrium allocation is then given by

$$
\begin{aligned}
& X^{*}=\sqrt{2 M_{1} / \lambda}, \\
& m_{x}^{*}=\sqrt{2 \lambda M_{1}}-\lambda x .
\end{aligned}
$$

\footnotetext{
${ }^{6}$ Strictly speaking, we should interpret this as the limit of a model with a discrete number of product and advertising markets. In the discrete model, all consumers of product $x$ are located in the advertising market $a=x$. Each firm $x$ only advertises in the online market $a=x$, supply is proportional to the number of consumers in the market, and as a consequence, the probability of a match is constant across firms. These results hold for any finite number of products and markets, and carry over to our continuous model.
} 
The equilibrium distribution of offline messages and the participating firms are hence identical to the single market case. It is useful to observe that, in the case of competition between symmetric, perfectly targeted media, the equilibrium price and media revenues would follow a similar pattern to the case of competition between symmetric offline media. In particular, the prices on the two media would be equal, and they would only depend on the total number of messages supplied to each market. The allocation of messages among firms would clearly differ, as each firm $x$ purchases the entire supply of messages in each market $a=x$.

However, competition between heterogeneous media has a different effect on the equilibrium prices and revenues, as we show in the next proposition.

\section{Proposition 8 (Equilibrium Prices)}

1. The equilibrium price on the offline medium is given by

$$
p^{*}=\lambda \exp \left(-M_{2}-\sqrt{2 \lambda M_{1}}\right) .
$$

2. The equilibrium prices on the online markets are given by

$$
p_{a}^{*}=\left\{\begin{array}{cc}
\exp \left(\lambda a-M_{2}-\sqrt{2 \lambda M_{1}}\right), & \text { for } a \leq X^{*} \\
\exp \left(-M_{2}\right), & \text { for } a>X^{*}
\end{array}\right.
$$

Consistent with intuition, the offline price $p^{*}$ is decreasing in $M_{2}$. This reflects the decline in each firm's willingness to pay for regular advertisements when an alternative, better targeted market is present. In other words, a targeted online market does not modify the composition of the offline market, but lowers the equilibrium profits. The prices in the online markets are initially increasing in $a$, and then constant. This reflects the allocation of messages offline, where relatively smaller firms buy a lower number of messages, and are willing to pay more for $M_{2}$ messages per capita online. Furthermore, the prices paid online are constant for all the firms that do not participate in the offline market. In other words, "niche" online markets, where customers of long tail firms are likely to be present, are not affected at all by media competition. In this sense, as emphasized by Anderson (2006), online advertising allows to reach new segments of the consumer population, which are distinct from the intended audience of the firms that actively advertise offline.

Up to this point, we have imposed no restrictions on the total supply of advertising space. We now seek to assess the implications of the consumer's relative exposure to online and offline advertisements. For this reason, we interpret the supply as the outcome of the consumer's time allocation decision. In particular, we assume each consumer spends a fraction $\beta$ of her time $M$ in the online medium. We then have $M_{1}=(1-\beta) M$ and 
$M_{2}=\beta M$ and, following (20), the number of firms active in the offline market is given by $X^{*} \triangleq \sqrt{2 M(1-\beta) / \lambda}$.

\section{Proposition 9 (Online Exposure)}

1. The equilibrium price in online markets $a>X^{*}$ is decreasing in $\beta$.

2. The equilibrium prices in online markets $a \leq X^{*}$ and in the offline market are decreasing in $\beta$ if and only if $\beta \leq 1-\lambda / 2 M$.

Thus, the equilibrium price of offline advertising does not vary monotonically with consumers' exposure to online media. When online exposure is low, the greater efficiency of online targeted messages reduces the marginal willingness to pay for offline advertising. This reduction more than offsets the price increase resulting from a lower supply of offline messages. In particular, Proposition 6 shows that when symmetric offline media are competing, the equilibrium price only depends on the total supply $M$. In this sense, for low $\beta$, the growth of online advertising markets is more detrimental for the price of an offline medium than the loss of market share to a traditional competitors. However, as the online exposure $\beta$ increases further, an important additional effect appears: a decrease in supply on the offline medium changes the identity - and hence the willingness to pay - of the marginal firm. As offline supply decreases, the largest, most valuable customers buy most of the advertising space. This effect, which is due to a reduction in supply, keeps the marginal returns high, and hence drives up the equilibrium price. This is radically different from the increase in asymmetry with a fixed supply we analyzed in Section 3 .

As the price offline increases, so do the online prices on the online markets $a \leq X^{*}$. For high levels of concentration $\lambda$, the change in the composition of the offline demand occurs faster, leading prices to increase in $\beta$. In particular, for $\lambda>2 M$, the equilibrium price is increasing in $\beta$ everywhere.

Recent work by Goldfarb and Tucker (2011) provides evidence supporting our findings on the substitution patterns between online and offline advertising. Goldfarb and Tucker (2011) analyze bidding data for "personal injury" Google keywords, and the prices paid by advertisers (law firms) in several locations. The variation in prices across locations is considerable, ranging from close to zero to over $\$ 50$ per click. Goldfarb and Tucker (2011)

exploit the exogenous variation introduced in the availability of offline advertising, due to the different state regulations on the ability for lawyers to contact clients by mail. In particular, they report advertising prices per click for search engine advertising are higher in the absence of an offline alternative. Furthermore, the substitution effect is stronger for law firms with smaller markets. This result resonates with the above results for media competition, which 
show how small and medium sized firms move away from the offline medium more quickly, as the degree of online exposure increases.

Finally, we examine the role of online exposure on the revenues (the number of matches) of different firms $x$.

Proposition 10 (Advertisers' Revenues) Assume $2 \lambda<M$.

1. The revenue of firms $x \geq X^{*}$ is increasing in $\beta$.

2. The revenue of firms $x \leq X^{*}$ is inverse- $U$ shaped in $\beta$.

The main implication of Proposition 10 is that online exposure benefits small and medium firms at the expense of larger firms. Indeed, as consumers spend more time online, the supply of messages offline decreases. As we pointed out, this implies the price increases and the set of participating firms becomes smaller. The larger firms therefore pay a higher price for their advertising offline. The smaller firms simply benefit from a larger online market, and their revenues are increasing in $\beta$. In consequence, our model predicts that the increase in the relative exposure of consumers to online media may be detrimental to the profits of larger firms, compared to small and medium ones. In particular, medium sized firms benefit unambiguously from the diffusion of online advertising. In other words, the predictions of our advertising model differ from those of Bar-Isaac, Caruana, and Cuñat (2011), who find that both large and small firms benefit from lower search costs, compared to medium-sized ones, as well as from those of Fleder and Hosanagar (2009), who find that recommender systems may benefit both widely popular and extremely niche sellers (but not intermediate ones).

When we consider imperfect targeting levels, our predictions are similar to those of the model with different degrees of concentration. In particular, the online market $a=0$ is a close substitute for the offline medium, as all consumer types are present (though with different intensities). As a result, the largest firms leave the offline medium and advertise exclusively online, in the largest markets $a$, leading to a decrease in the price of the offline medium. This effect is somewhat mitigated if, as a robustness check, the online market has a smaller user base. With some modification due to this more complicated setup, the analysis of competition between offline media of different sizes extends to the case of imperfect targeting.

\section{Concluding Remarks}

We developed a novel model to understand the implications of targeting in advertising markets. The model provided a framework for the systematic analysis of the trade-offs that arise 
due to changes in the targeting technology. We adopted a hierarchical framework to rank products and advertising markets of different sizes. We explored in particular the tension between competition and value extraction that appears as the targeting ability of the various media improves. In terms of welfare, our analysis highlights the strong benefits due to targeting in a competitive environment. At the same time, we caution that the source of targeting, namely large amounts of information about consumers, may often lead to highly concentrated market structures, as it is the case in search and social networks with Google and Facebook, respectively.

We discussed earlier the robustness of our findings to alternative matching structures and targeting technologies. Our analysis identifies conditions that extend our results beyond the exponential framework adopted here. As these conditions are not specific to the case of display advertising, or broadcasting, it follows that our model can provide insight into the effects of detailed users information in the hands of internet content providers, and on the profitability of IP address tracking.

The analysis we have presented is the outcome of a number of modeling choices which constrain the scope of our results in some directions. We now conclude by discussing several directions for future research that relax some of our assumptions.

Market Power The price of advertising was determined in a competitive equilibrium model. While this is a natural benchmark, it is of interest to consider the pricing of advertising in strategic environments. In the working paper version, Bergemann and Bonatti (2010), we investigate the equilibrium pricing when each advertising market is populated with a small number of publishers, each one maximizing his revenues. Publishers compete à la Cournot, and determine the number of messages to supply to the market. We establish that already with a small number of publishers, the Nash equilibrium yields the competitive equilibrium outcome analyzed here. Clearly, in a context where publishers have market power, extending our model to incorporate the auctions for keywords in the sponsored search environment, or the emerging ad exchange model, might also offer valuable additional insights.

In our model, the advertisers were competing for messages but they were not competing for consumers. In other words, competition among firms for advertising messages did not interact with their competition in the product market. A natural next step therefore might be to enrich the current model with advertisers that are directly competing in the product markets. The equilibrium price for advertising, in particular in highly targeted markets, may then interact with the intensity of competition in the product market. 
Multi-Homing We assumed that each consumer is only present in one advertising market. A mathematically equivalent interpretation is that each consumer of product $x$ visits a specific medium $a$ following the distribution $s(a \mid x)$. We could further weaken the singlehoming assumption by interpreting $s(a \mid x)$ as the amount of time during which a consumer of product $x$ is exposed to the advertising market $a$. The only difference that emerges with this modelling choice is that messages in two distinct advertising markets $a$ and $a^{\prime}$ now act as substitutes with respect to the probability of generating a sale from a customer. In other words, with random single-homing, the returns from messages in two different markets are additive; in contrast, with the multi-homing interpretation, they are subadditive. Now, the only change in the demand for advertising occurs due to the substitutability property. With a continuum of advertising markets, the demand for advertising by firm $x$ in advertising market $a$ is simply discounted by a constant factor equal to the probability that the consumer has already been successfully contacted in any one of the other markets. Importantly, this discounting factor is going to be smaller for larger firms, as they send more messages across the advertising markets. Relative to the current equilibrium in the many markets model, the net effect is that the demand for advertising is less dispersed, and the differences in market sizes are attenuated. Large firms send more messages, and hence are more likely to have already attracted the consumer on some other market. But the qualitative analysis and the impact of targeting remain unchanged. In particular, as targeting becomes more prevalent, the effect of substitutions weakens and, more importantly, becomes more uniform across firms as differences in market size play a lesser role.

Endogenous Consumer Locations Finally, the distribution of consumers across advertising markets was given exogenously. A natural next step would be to extend the model to consumers whose location choice in advertising outlets reflects an optimization decision. Along the lines of Anderson and Coate (2005), each medium provides content and advertising for the consumer. While content has positive value to the consumers, advertising has negative value. In the spirit of the current model, the disutility of advertising would be increasing in the distance of the advertisement message from the interest of the reader. In such a market, as shown in Armstrong (2006), if publishers have market power and consumers single home on advertising markets, we would expect the total supply of advertising to be inefficiently low and the equilibrium prices to be above the competitive level. This "competitive bottleneck" framework could then deliver important insights into the effects of competition between a general interest traditional medium, such as the New York Times or the Wall Street Journal, and a general interest portal, such as Google or Yahoo!, that can personalize the distribution of advertisements through information about the consumer. 


\section{Appendix}

Proof of Proposition 1. The average probability of a match, which is equal to the total fraction of consumers reached, is given by

$$
W(\lambda, M)=\int_{0}^{X^{*}} s_{x}\left(1-e^{-m_{x}^{*}}\right) d x=1-\frac{1+\sqrt{2 M \lambda}}{e^{\sqrt{2 M \lambda}}},
$$

which is increasing in $\lambda$.

Proof of Proposition 2. (1.)-(4.) The comparative statics results can be derived directly by differentiating expressions (10), (11), and (12) in the text.

(5.) The total expenditure of firm $x \leq X^{*}$ is given by

$$
p^{*} m_{x}^{*}=\lambda e^{-\sqrt{2 \lambda M}}(\sqrt{2 \lambda M}-\lambda x)
$$

and the total number of consumers reached is

$$
s_{x}\left(1-e^{-m_{x}^{*}}\right)=\lambda e^{-\lambda x}\left(1-e^{\lambda x-\sqrt{2 \lambda M}}\right) .
$$

Therefore, the price paid by firm $x$ per consumer reached is given by

$$
\frac{p^{*} m_{x}^{*}}{s_{x}\left(1-e^{\left.-m_{x}^{*}\right)}\right.}=\frac{\sqrt{2 \lambda M}-\lambda x}{e^{\sqrt{2 \lambda M}-\lambda x}-1}=\frac{z}{e^{z}-1},
$$

which is decreasing in $z$ (with $z=\sqrt{2 \lambda M}-\lambda x$ ), and therefore increasing in $x$. It is also decreasing in $\lambda$ if $x<\sqrt{M / 2 \lambda}$ (which represents the median active firm).

Proof of Proposition 3. The average probability of a match now takes into account the fraction of consumers reached in the exterior market as well as in the interior markets. It is given by,

$$
W(\lambda, \gamma, M)=\int_{0}^{\infty} \int_{a}^{X_{a}^{*}} s_{a, x}\left(1-e^{-m_{a, x} / s_{a}}\right) d a d x+\int_{0}^{X_{0}^{*}} s_{x, 0}\left(1-e^{-m_{x, 0} / s_{0}}\right) d x
$$

where $m_{a, x}^{*}$ is given by (17) in the text. Therefore, we obtain

$$
W(\lambda, \gamma, M)=1-\frac{1+\sqrt{2 M(\lambda+\gamma)}}{e^{\sqrt{2 M(\lambda+\gamma)}}}
$$

which is increasing in $\lambda$ and $\gamma$. 
Proof of Proposition 4. (1.) Consider firms $x \geq \bar{X}=\bar{X}(\gamma)=\sqrt{2 M /(\gamma+\lambda)}$. The number of matches generated by firm $x$ on market $a$ are given by

$$
s_{x, a}\left(1-e^{-m_{x, a} / s_{a}}\right)=\lambda \gamma e^{-(\lambda+\gamma) x+\gamma a}\left(1-e^{-(\gamma+\lambda)\left(X_{0}^{*}-(x-a)\right)}\right)
$$

Integrating over markets, we obtain

$$
\begin{aligned}
W_{x} & =\int_{x-X_{0}^{*}}^{x}\left(\lambda \gamma \frac{e^{a \gamma}}{e^{x \lambda} e^{x \gamma}}-\lambda \frac{\gamma}{e^{X_{0}^{*} \lambda} e^{X_{0}^{*} \gamma} e^{a \lambda}}\right) d a \\
& =\lambda e^{-\lambda x}+e^{-\lambda x} e^{-X_{0}^{*} \gamma}\left(\gamma\left(e^{-X_{0}^{*} \lambda}-1\right)-\lambda\right) .
\end{aligned}
$$

Now consider the derivative of $W_{x}$ with respect to $\gamma$ :

$$
\frac{\partial W_{x}}{\partial \gamma} \propto \sqrt{2} M \lambda-(\sqrt{M(\lambda+\gamma)}-M \gamma / \sqrt{2})\left(1-\exp ^{-\lambda \sqrt{2 M /(\lambda+\gamma)}}\right)
$$

Note that when $\gamma=0$, this expression is equal to $e^{-b}+b-1 \geq 0$, with $b=\lambda M$. As $\gamma \rightarrow \infty$, we obtain $\partial W_{x} / \partial \gamma \rightarrow \infty$. Finally, consider the derivative of $W_{x}$ with respect to $\bar{X}$ (and let $\left.\gamma=2 M / \bar{X}^{2}-\lambda\right)$. Its sign depends on:

$$
\frac{\partial W_{x}}{\partial \bar{X}} \propto-\left(2\left(e^{-\bar{X} \lambda}-1\right)(\bar{X}-M)+\bar{X}^{2} \lambda\left(1+e^{-\bar{X} \lambda}\right)\right)
$$

We only need to verify that this expression is monotone in $\bar{X}$, so its sign cannot change twice. Indeed, differentiating with respect to $\bar{X}$, we obtain

$$
\begin{aligned}
\frac{\partial^{2} W_{x}}{(\partial \bar{X})^{2}} & \propto \bar{X}^{2} \lambda^{2} e^{-\bar{X} \lambda}-2 \bar{X} \lambda-2 e^{-\bar{X} \lambda}+2-2 M \lambda e^{-\bar{X} \lambda} \\
& =\left(z^{2}-2-2 M \lambda\right) e^{-z}+2-2 z<0, \text { with } z=\bar{X} \lambda .
\end{aligned}
$$

Therefore, the number of matches is always increasing in $\gamma$ for small firms $x>\bar{X}$.

(2.) All firms $x \leq \bar{X}$ advertise on markets $a \in[0, x]$. Therefore the number of matches generated by firm $x$ is given by

$$
W_{a, x}= \begin{cases}\lambda \gamma e^{-(\lambda+\gamma) x} e^{\gamma a}\left(1-e^{-(\gamma+\lambda)(\bar{X}-(x-a))}\right), & \text { if } \quad 0<a<x, \\ \lambda e^{-(\lambda+\gamma) x}\left(1-e^{-(\gamma+\lambda)(\bar{X}-x)}\right), & \text { if } \quad a=0 .\end{cases}
$$


Integrating across markets $a \in[0, x]$, we obtain

$$
W_{x}=\lambda e^{-\lambda x}-\frac{\gamma\left(1-e^{-\lambda x}\right)+\lambda}{e^{\sqrt{2 M(\gamma+\lambda)}}} .
$$

The derivative with respect to $\gamma$ is given by

$$
\left.\frac{\partial W_{x}}{\partial \gamma} \propto\left(e^{-x \lambda}-1\right)\right)(\sqrt{2 M(\lambda+\gamma)}-M \gamma)+M \lambda
$$

Therefore, we obtain $\partial W_{x} / \partial \gamma>0$ if and only if $e^{-\lambda x}>1-M \lambda /(\sqrt{2 M(\lambda+\gamma)}-M \gamma)$. This defines a threshold firm $\bar{x}=\bar{x}(\gamma)$ such that all $x \leq \bar{x}$ generate a larger number of matches as $\gamma$ increases.

(3.) When $\sqrt{M(\lambda+\gamma)}-M \gamma<0$, we obtain $\partial W_{x} / \partial \gamma>0$ for all $x$ and $\gamma$. This is the sufficient condition provided in the text.

Proof of Proposition 5. (1.)-(4.) These statements follow from differentiation of expressions (15), (16), and (17) in the text.

Proof of Proposition 6. From the first order conditions for firm $x$, we obtain

$$
1-f_{i, x}=e^{-m_{i, x}}=e^{\lambda x} \frac{p_{i}}{\lambda\left(1-f_{j, x}\right)}, i \neq j
$$

It follows that in equilibrium we must have $p_{1}=p_{2}=p$, and that the sum of the demands satisfies $m_{1, x}+m_{2, x}=\ln \frac{\lambda}{p}-\lambda x$. We join the market clearing conditions for 1 and 2 :

$$
\int_{0}^{X}\left(m_{1, x}+m_{2, x}\right) d x=M_{1}+M_{2}
$$

and the results follow as in the single-homing case.

Proof of Proposition 7. The first order conditions are:

$$
\begin{aligned}
\lambda_{1} e^{-\lambda_{1} x}\left(1-\delta\left(1-e^{-m_{2, x} / \delta}\right)\right) e^{-m_{1, x}}-p_{1} & =0 \\
\lambda_{1} e^{-\lambda_{1} x} e^{-m_{1, x}}\left(1-e^{-m_{2, x} / \delta}\right)-p_{2} & =0
\end{aligned}
$$

Solving for $m_{1, x}$ and $m_{2, x}$, and simplifying, we obtain

$$
\begin{aligned}
& m_{1, x}=\ln \frac{\lambda(1-\delta)}{p_{1}-\delta p_{2}}-\lambda x \\
& m_{2, x}=m_{2}=\delta \ln \frac{p_{1}-\delta p_{2}}{p_{2}(1-\delta)}, \text { for } x \in[0, X]
\end{aligned}
$$


For all firms $x \in[X, Z]$, we have $m_{1, x}=0$ and $m_{2, x}=\delta\left(\ln \lambda / p_{2}-\lambda x\right)$ as in the singlehoming case. Since by construction, the marginal firm $X$ satisfies $m_{1, X}=0$, we have $(1-\delta) \lambda \exp (-\lambda X)=p_{1}-\delta p_{2}$. Similarly, we have $m_{2, Z}=0$, and so $\lambda \exp (-\lambda Z)=p_{2}$. We can now write the market clearing conditions as follows:

$$
\begin{aligned}
\int_{0}^{X} m_{1, x} d x & =\int_{0}^{X} \lambda(X-x) d x=M_{1}, \\
X m_{2, x}+\int_{X}^{Z} m_{2, x} d x & =X \delta \lambda(Z-X)+\int_{X}^{Z} \delta \lambda(Z-x) d x=\delta M_{2} .
\end{aligned}
$$

Therefore $X=\sqrt{2 M_{1} / \lambda}$ and $Z=\sqrt{2\left(M_{1}+M_{2}\right) / \lambda}$ which implies that $p_{1}=\delta \lambda e^{-\sqrt{2\left(M_{1}+M_{2}\right) \lambda}}+$ $(1-\delta) \lambda e^{-\sqrt{2 M_{1} \lambda}}$, and $p_{2}=\lambda e^{-\sqrt{2\left(M_{1}+M_{2}\right) \lambda}}$.

Proof of Proposition 8. The price offline is equal to $\lambda \exp \left(-\lambda X^{*}\right)$, where $X^{*}$ is the marginal firm characterized in (20). The prices offline follow from substitution of (21) into (18) and (19).

Proof of Proposition 9. (1.) The equilibrium price $p_{a}$ for $a>X^{*}$ is decreasing in $M_{2}$ and hence in $\beta$.

(2.) The sign of the derivative of $p$ and $p_{a}$ for $a \leq X^{*}$ depends on the term

$$
\beta M+\sqrt{2 \lambda(1-\beta) M}
$$

which is decreasing in $\beta$ everywhere if $\lambda>2 M$, or increasing in $\beta$ for $\beta \leq 1-\lambda / 2 M$.

Proof of Proposition 10. (1.) Small firms $x>X^{*}$ buy $s_{x} \beta M$ messages at a price of $e^{-\beta M}$ online. Their profits are given by $\pi_{x}=s_{x}\left(1-e^{-\beta M}-\beta M e^{-\beta M}\right)$, which is increasing in $\beta$.

(2.) Large firms $x<X^{*}$ buy $s_{x} \beta M$ messages at a price of $e^{-\beta M} e^{-m_{x}}$ online and $\sqrt{2 \lambda(1-\beta) M}-\lambda x$ messages offline at a price of $\lambda e^{-\beta M-\sqrt{2 \lambda(1-\beta) M}}$. Therefore profits are given by $\pi_{x}=\lambda e^{-\lambda x}-\lambda e^{-z}(1+z-\lambda x)$, with $z=\beta M-\sqrt{2 \lambda(1-\beta) M}$. Notice that the derivative with respect to $z$ is given by

$$
\frac{\partial \pi_{x}}{\partial z}=\lambda e^{-z}(z-x \lambda)
$$

Therefore, if $\sqrt{2 \lambda M}<M$, we obtain that $z-x \lambda \geq \sqrt{2 \lambda M}-\lambda x>0$ for all $x<X^{*}$, and therefore the number of matches is increasing in $z$ (hence inverse-U shaped in $\beta$ ) for all $x \leq X^{*}$. 


\section{References}

Ambrus, A., And M. Reisinger (2006): "Exclusive vs Overlapping Viewers in Media Markets," Discussion paper, University of Munich.

Anderson, C. (2006): The Long Tail. Hyperion New York.

Anderson, S., And S. CoAte (2005): "Market provision of broadcasting: A welfare analysis," Review of Economic Studies, pp. 947-972.

Anderson, S., and A. De Palma (2009): "Information congestion," RAND Journal of Economics, 40(4), 688-709.

Armstrong, M. (2006): "Competition in Two-Sided Markets," RAND Journal of Economics, 37, 668-691.

Athey, S., And G. Ellison (2011): "Position Auctions with Consumer Search," Quarterly Journal of Economics, forthcoming.

Athey, S., And J. Gans (2010): "The Impact of Targeting Technology on Advertising Markets and Media Competition," American Economic Review Papers and Proceedings, $100,608-613$.

BAGWELL, K. (2007): "The economic analysis of advertising," Handbook of Industrial Organization, 3, 1701-1844.

Bar-IsaAc, H., G. Caruana, and V. Cuñat (2011): "Search, Design and Market Structure," American Economic Review, forthcoming.

Bergemann, D., And A. Bonatti (2010): "Targeting in Advertising Markets: Implications for Offline Vs. Online Media," Discussion paper, Cowles Foundation for Research in Economics, Yale University.

Butters, G. (1977): "Equilibrium distributions of sales and advertising prices," The Review of Economic Studies, 44(3), 465-491.

Chandra, A., and U. Kaiser (2010): "Targeted Advertising in Magazine Markets," Discussion paper, University of British Columbia.

Chen, Y., And C. He (2006): "Paid placement: Advertising and search on the internet," Discussion paper, University of Colorado at Boulder. 
De Cornière, A. (2010): "Targeted Advertising with Consumer Search: an Economic Analysis of Keywords Advertising," Discussion paper, Paris School of Economics.

Dukes, A. (2004): "The Advertising Market in a Product Oligopoly," The Journal of Industrial Economics, 52(3), 327-348.

Edelman, B., M. Ostrovsky, and M. Schwarz (2007): "Internet advertising and the generalized second-price auction: Selling billions of dollars worth of keywords," American Economic Review, 97(1), 242-259.

Esteban, L., A. Gil, and J. Hernandez (2001): "Informative advertising and optimal targeting in a monopoly," The Journal of Industrial Economics, 49(2), 161-180.

Ferrando, J., J. Gabszewicz, D. Laussel, and N. Sonnac (2004): "Two-Sided Network Effects and Competition: An Application to Media Industries," in Conference on The Economics of Two-Sided Markets, Toulouse.

Fleder, D., And K. Hosanagar (2009): "Blockbuster culture's next rise or fall: The impact of recommender systems on sales diversity," Management Science, 55(5), 697-712.

Goldfarb, A., And C. Tucker (2011): "Search Engine Advertising: Channel Substitution when Pricing Ads to Context," Management Science, forthcoming.

Iyer, G., D. Soberman, and M. Villas-Boas (2005): "The Targeting of Advertising," Marketing Science, 24(3), 461.

Johnson, J. (2010): "Targeted advertising and advertising avoidance," Discussion paper, Johnson Graduate School of Management, Cornell University.

Johnson, J., and D. Myatt (2006): "On the Simple Economics of Advertising, Marketing, and Product Design," American Economic Review, 96(3).

Levin, J., And P. Milgrom (2010): "Online Advertising: Heterogeneity and Conflation in Market Design," American Economic Review Papers and Proceedings, 100, 603-607.

Rutz, O., And R. Bucklin (2011): "From generic to branded: A model of spillover dynamics in paid search advertising," Journal of Marketing Research, forthcoming.

VAN ZANDT, T. (2004): "Information overload in a network of targeted communication," RAND Journal of Economics, 35(3), 542-560.

VARIAN, H. (2007): "Position auctions," International Journal of Industrial Organization, $25(6), 1163-1178$. 